\title{
COMPETENCIAS PROFESIONALES REQUERIDAS EN LA FORMACIÓN DE LOS FISIOTERAPEUTAS COLOMBIANOS
}

\author{
${ }^{1}$ Aminta Stella Casas Sánchez, ${ }^{2}$ Paula Camila Ramírez Muñoz \\ ${ }^{1}$ Magíster en Salud Pública, Universidad de Antioquia. \\ Docente Escuela de Fisioterapia Universidad Industrial de Santander, Bucaramanga, Colombia \\ ${ }^{2}$ Magíster en Epidemiología, Universidad Industrial de Santander. \\ Docente Escuela de Fisioterapia Universidad Industrial de Santander, Bucaramanga, Colombia
}

Autor responsable de correspondencia: Aminta Stella Casas Sánchez

Correo electrónico: ascasas@uis.edu.co

RESUMEN

Revisar las competencias profesionales requeridas en la formación de los fisioterapeutas colombianos. Se realizó una revisión documental y análisis de los lineamientos internacionales y nacionales acerca de las competencias requeridas en la formación de fisioterapeutas en colombia, que les permitan responder pertinentemente a las necesidades del país. Agremiaciones profesionales internacionales y nacionales proponen competencias comunes con otros profesionales de salud: ética, responsabilidad profesional, comunicación y trabajo en equipo. Además, se proponen competencias específicas que orientan su actuar en las áreas de promoción de la salud, prevención primaria, secundaria y terciaria de la enfermedad, en diversos campos de actuación profesional, que permitan dar una respuesta social dirigida hacia la persona, la familia y la comunidad. La Fisioterapia es una profesión del área de la salud enfocada al estudio y comprensión del movimiento corporal humano; el ejercicio profesional se orienta al mantenimiento, optimización y potencialización del movimiento, así como a la prevención de sus alteraciones y a la rehabilitación integral de las personas. El fisioterapeuta requiere desarrollar competencias que le permitan ejercer su rol profesional y aportar en equipos inter y multidisciplinarios, contribuyendo a mejorar la calidad de vida de individuos y comunidades. [Casas AS, Ramírez PC. Competencias profesionales requeridas para la formación de los fisioterapeutas colombianos. Ustasalud. 2015;14:48-52]

Palabras clave: Profesional de salud, fisioterapia, educación basada en competencias, sistemas de salud.

\section{PROFESSIONAL SKILLS REQUIRED IN THE TRAINING OF PHYSICAL THERAPISTS IN COLOMBIA}

\begin{abstract}
To review the professional skills required in professional education for physical therapists in Colombia. A literature review and an analysis of international and national lineaments of required skills in professional education for physical therapists in Colombia that allow them to respond effectively to the needs of the country were performed. International and national organizations and professionals propose skills that are common to other health professionals: ethics, professional responsibility, communication and teamwork. Furthermore, specific competencies are proposed that guide their action in the areas of health promotion, primary, secondary and tertiary prevention of the disease (or 'diseases'?) in diverse professional fields, that allow physical therapists to give in a social response addressed to the person, family and community. Physical therapy is a profession of the health area focused on the study and understanding of human body movement. The professional duty is not only oriented to the maintenance, optimization and potentiation of the movement, but also to the prevention of its deviation and the integral rehabilitation of the person. The physical therapist requires the developing of skills and competencies that allow them to exercise their professional role and contribute to inter- and multidisciplinary teams; this helps to improve the quality of life of individuals and communities.
\end{abstract}

Keywords: Health personnel, physical therapists, competency -based education, Health Systems.

Recibido para publicación: 18 de mayo del 2015. Aceptado para publicación: 23 de agosto de 2015.

\section{INTRODUCCIÓN}

Entendida la Salud como un estado de completo bienestar físico, mental y social y no solamente la ausencia de enfermedad, los fisioterapeutas proveen servicios a las personas y poblaciones para mantener y restaurar el máximo movimiento y habilidad funcional a lo largo de toda la vida. La práctica fisioterapéutica incluye la provisión de servicios en circunstancias en las que el movimien- to y la función están amenazados por el proceso de envejecimiento, desórdenes o enfermedades, siendo el movimiento funcional fundamental para lo que significa ser saludable.

Para el ejercicio profesional se requiere desarrollar durante la formación, tanto competencias específicas que le permitan ejercer el rol profesional propio, como competencias generales comunes con los demás profesionales de la salud, entendiendo que 
la atención en salud debe centrarse en el pacientecliente como ser integral.

A partir de la revisión de literatura sobre el tema, se han identificado competencias afines emanadas por diversas organizaciones internacionales, así como, competencias del orden nacional en coherencia con los lineamientos impartidos por la Comisión del Talento Humano en Salud del Ministerio de Protección Social.

\section{LINEAMIENTOS INTERNACIONALES PARA LA PRÁCTICA DE LA FISIOTERAPIA}

El ejercicio de la fisioterapia tiene que ver con la identificación y maximización de la calidad de vida y la potencialización del movimiento funcional en los ámbitos de promoción, prevención, mantenimiento, intervención, habilitación y rehabilitación. Esto abarca el bienestar físico, psicológico, emocional y social, e implica la interacción entre el terapeuta físico, los pacientes o clientes, familias, cuidadores, otros proveedores de atención de salud y las comunidades ${ }^{1,2,3}$.

Según lo planteado por la Comisión de Acreditación en la Educación de Terapia Física (CAPTE, por su sigla en inglés), el fisioterapeuta debe promover la práctica regular de actividad física y prevenir la discapacidad, mediante el conocimiento e intervención oportuna de los factores de riesgo, además de participar con liderazgo en organizaciones comunitarias y de servicio voluntario, influyendo en los procesos legislativos y políticos que redunden en mejorar la salud y el bienestar de la sociedad ${ }^{4}$.

La Guía del componente clínico de educación para el pregrado de la fisioterapia desarrollada por la Confederación Mundial de Fisioterapia, WCPT por sus siglas en Inglés ${ }^{1}$, señala la necesidad de garantizar las siguientes oportunidades durante el proceso formativo:

- Integrar conocimientos, habilidades y comportamientos profesionales y aplicarlos en la Clínica.

- Aprender a través de la práctica, la experiencia y la reflexión.

- Mejorar habilidades clínicas para el proceso de atención del paciente.

- Mejorar las habilidades de comunicación en todos los niveles que permitan demostrar un comportamiento profesional apropiado, experiencia de socialización profesional e interprofesional.

El desarrollo de estas habilidades favorece un alto sentido de responsabilidad y el ejercicio autónomo de la profesión ${ }^{1}$.
En coherencia con los lineamientos de la WCPT, organizaciones de diversos países han elaborado las normas que rigen la profesión: el Consejo de Profesiones de Salud y Asistencia (HCPC, por sus siglas en inglés), en el Reino Unido, está encargado de regular la práctica de las profesiones de salud, en las que está incluida la Fisioterapia; en el 2013 publicó la versión más reciente de los estándares para la práctica del fisioterapeuta. Las normas contenidas en el documento describen lo que un estudiante debe conocer, comprender y ser capaz de hacer una vez haya concluido su formación.

Los estándares contemplan el desarrollo de una práctica segura, donde se reconozcan los límites profesionales, el cumplimiento de los aspectos éticos y legales, la comprensión de la necesidad de respetar y defender los derechos, la dignidad, los valores y la autonomía de los usuarios del servicio, incluyendo su rol en el diagnóstico, el proceso terapéutico y en el mantenimiento de la salud y el bienestar. Ser conscientes del impacto de la cultura, la igualdad y la diversidad en la práctica, adaptándola a las necesidades de diferentes grupos e individuos, manteniendo una comunicación efectiva con los usuarios y colegas ${ }^{5}$.

La declaración Europea Referencial de Formación en Fisioterapia (European Physiotherapy Benchmark Statement) describe la naturaleza y estándares de los programas de fisioterapia impartidos por las instituciones de educación superior en Europa, definiendo el umbral mínimo para los estudiantes que se gradúan en fisioterapia, dentro de los que se incluyen: habilidades genéricas y profesionales específicas, bases científicas de la fisioterapia, contexto de la prestación del servicio, así como competencias comunes con otros profesionales de la salud, entre las que se incluyen: autonomía y responsabilidad profesional, trabajo interdisciplinario, comunicación efectiva con clientes y cuidadores; comprensión de su rol como cuidador de salud y de políticas gubernamentales para la provisión de servicios de salud ${ }^{6}$.

Por su parte los estándares en Australia incluyen un enfoque holístico que abarca la prevención, diagnóstico, manejo del dolor y desórdenes del movimiento, así como la optimización de la función y bienestar de individuos y poblaciones. Los estándares planteados por el Consejo Australiano de Fisioterapia incluyen criterios y elementos relacionados con la comunicación efectiva, acceso, interpretación y aplicación de la información, interpretación y análisis de los hallazgos de la evaluación, intervención profesional efectiva en diversos campos de actuación profesional en los que se incluyen organiza- 
ciones de salud, escuelas, escenarios deportivos, laborales y comunidades ${ }^{7}$.

De otra parte, se reconoce la importancia del trabajo con otros profesionales de salud, para dar una respuesta integrada y coherente a las necesidades del cliente/familia/ población, lo que se ha denominado "interprofesionalidad" 8,9 . El panel de expertos de educación interprofesional, hace referencia a cuatro tipos de competencias interprofesionales: valores/ética, roles/responsabilidades, comunicación interprofesional y trabajo en equipo ${ }^{9}$; siendo la interacción continua y el intercambio de conocimientos entre profesionales lo que garantiza la más alta calidad de la atención ${ }^{8,9}$.

En este sentido la Alianza Mundial de Profesiones de Salud (The World Health Professions Alliance WHPA) de la cual hace parte la WCPT, señala que la práctica efectiva de colaboración interprofesional puede conducir a mejorar el acceso a las intervenciones de salud, mayor participación del individuo y la familia en la toma de decisiones, uso eficiente de recursos, reducción de la incidencia y la prevalencia de la discapacidad, en particular la asociada con las enfermedades no transmisibles, dado que se puede abarcar todo el ciclo de atención: promoción de la salud, prevención de la enfermedad, curación y rehabilitación; adicionalmente el aumento de satisfacción en el trabajo, con la reducción del estrés y el agotamiento de los profesionales de la salud ${ }^{10}$.

La Organización Mundial de la Salud - OMS y la Organización Panamericana de la Salud - OPS, a través del documento Llamado a la acción de Toronto ${ }^{11}$ plantean como un desafío la formación de los trabajadores de la salud, de manera que respondan a un modelo de atención universal, equitativo y de calidad, que sirva a las necesidades de la población. Igualmente, a nivel de la región de las Américas en relación con el recurso humano en salud, se evidencia la necesidad de reformar la educación de los profesionales para apoyar a los sistemas de salud basados en la atención primaria, avanzando hacia la cobertura universal, sin demeritar la calidad de los servicios de salud ${ }^{12}$.

\section{LINEAMIENTOS PARA EL EJERCICIO Y LA FORMACIÓN DE LA FISIOTERAPIA EN COLOMBIA}

El ejercicio de la profesión de fisioterapia en Colombia se rige tanto por los principios generales formulados por la WCPT, como por los expresados en las Leyes 1164 de 2007 y 528 de 1999. La Ley 1164 establece las disposiciones relacionadas con los procesos de planeación, formación, vigilancia y control del ejercicio profesional, desempeño y ética del talento humano del área de la salud, mediante la articulación de los diferentes actores que intervienen en estos procesos ${ }^{13}$. La Ley 528 reglamenta el ejercicio de la profesión de fisioterapia en Colombia y dicta normas en materia de ética profesional y otras disposiciones ${ }^{14}$.

Mediante la Ley 1164 de 2007, artículo 8, se creó el Observatorio de Talento Humano en Salud (OTHS), el cual se orienta por los principios definidos en el Llamado a la Acción de Toronto 2006-2015: Hacia una década de los Recursos Humanos en Salud para las Américas"11,13. Las salas temáticas creadas a partir de esta normatividad son grupos inter y transdisciplinarios de profesionales expertos o investigadores que participan en asuntos específicos de talento humano en salud. La Sala Temática "Competencias Profesionales", se creó como espacio de participación, para que los profesionales aporten desde su conocimiento, experiencia y perspectiva de profesión, al proceso de actualización del perfil y las competencias de los profesionales de la salud.

El Grupo interinstitucional para elaboración de propuestas de perfiles y competencias profesionales básicas en salud inició en el 2009 un proceso de revisión y actualización de perfiles y competencias de los profesionales de la salud. El Grupo describe las competencias como "la conjunción de conocimientos, destrezas, aptitudes y actitudes que deben desarrollar los profesionales de la salud para atender adecuadamente las necesidades de salud de la población en el contexto del Sistema General de Seguridad Social en Salud"15.

Adicionalmente, se hace una diferenciación entre competencias transversales y específicas, siendo las primeras las competencias que las profesiones de la salud comparten en varias áreas o aspectos que hacen posible el trabajo interprofesional. De acuerdo con los lineamientos establecidos, se definieron seis competencias transversales: profesionalismo y ética, comunicativas, investigativas, administrativas y de gestión y razonamiento profesional, salud pública y gestion social. Las competencias específicas corresponden a las actuaciones propias de cada profesión.

El perfil profesional y competencias del fisioterapeuta en Colombia es uno de los 13 documentos preliminares de perfiles y competencias de los profesionales de la salud en el país, el cual es el resultado del trabajo conjunto de un grupo de profesionales que representan la Asociación Colombiana de Fisioterapia (ASCOFI), el Colegio Colombiano de Fisioterapeutas (COLFI) y la Asociación Colombiana de Estudiantes de Fisioterapia (ACEFIT) ${ }^{16}$. 
El documento recoge lineamientos internacionales del ejercicio profesional, así como los retos a los que debe enfrentarse en el contexto actual: 1) la salud mental de las poblaciones y las enfermedades crónicas no transmisibles, 2) envejecimiento poblacional, 3) las condiciones de salud de la mujer y sus relaciones con la familia, la salud de los niños, niñas y adolescentes, 4) la prevención de las discapacidades en el adulto en el futuro, 5) la fisioterapia familiar y comunitaria ${ }^{17}$. Asumir estos retos implica el contar con profesionales altamente competentes, líderes en gestión política, social, con competencias en comunicación individual y grupal, capacidad investigativa, con una formación sólida que permita contribuir a la solución de la problemática social y de salud en Colombia.

Las competencias específicas fueron definidas teniendo en cuenta los escenarios, ámbitos y territorios donde se desarrolla el ejercicio profesional del fisioterapeuta: clínica, actividad física y deporte, salud y trabajo, educación. Este documento se puede consultar en la sección salud, profesiones y ocupaciones, disponible en la página web del Ministerio de Salud y Protección Social.

Los documentos disponibles sobre competencias formuladas por los programas de fisioterapia acreditados en el país plantean dos grandes grupos de competencias: clínicas de rehabilitación, y promoción y prevención de la enfermedad; el primer grupo incluye competencias para manejar oportunamente la enfermedad: examen, evaluación, diagnóstico, pronóstico e intervención y seguimiento, mientras el segundo abarca lo relacionado con favorecer estilos de vida saludables por medio de la potenciación del movimiento corporal humano en colectivos sanos y el desarrollar acciones encaminadas a la detección temprana y control de factores de riesgo. Adicionalmente, se incluyen las competencias de investigación, que propenden por el desarrollo de un ejercicio profesional basado en la mejor evidencia científica, así como por la renovación y transformación del conocimiento profesional y de competencias de administración y gestión de servicios de salud ${ }^{17-21}$.

A partir de lo revisado podemos concluir que la formación del recurso humano en salud debe articularse con las características y necesidades en salud de la población, con los estándares aceptados internacionalmente y con los requerimientos del Sistema de Seguridad Social en Salud del país. En Colombia, de acuerdo con la Ley 1438 de 2011 se hace indispensable el desarrollo de competencias asociadas a la educación y promoción de la salud, atención primaria en salud - APS; procesos de gestión y coor- dinación de programas e intervenciones de carácter familiar y comunitario. Además se requiere orientar la formación con respecto a las culturas médicas tradicionales de los grupos étnicos y la implementación del enfoque intercultural ${ }^{22}$.

Corresponde a los programas de formación de fisioterapeutas y de salud en general, la formación de profesionales que, como parte del equipo interdisciplinario, aporten en el bienestar y calidad de vida de la población colombiana, especialmente a los grupos más vulnerables, atendiendo a los principios de equidad, justicia social y ética profesional.

\section{REFERENCIAS}

1. World Confederation for Physical Therapy (WCPT). Guideline for physical therapist professional entry-level education. Londres, WCPT; 2013. (Acceso 24 de junio de 2015). Recuperado a partir de: http://www.wcpt.org/sites/wcpt. org/files/files/Guideline_PTEducation_complete.pdf

2. World Confederation for Physical Therapy (WCPT). Policy statements. Londres, WCPT; 2013. (Acceso 24 de junio de 2015). Recuperado a partir de: http://www.wcpt.org/sites/ wcpt.org/files/files/WCPT_Policy_statements_2013.pdf

3. World Confederation for Physical Therapy (WCPT). Policy statements: Description of physical therapy. Londres, WCPT; 2013. (Acceso 24 de junio de 2015). Recuperado a partir de: http://www.wcpt.org/sites/wcpt.org/files/files/ PS_Description_PT_Sept2011_FORMATTED_edit2013.pdf

4. Commission on Accreditation in Physical Therapy Education (CAPTE). Evaluative Criteria PT Programs. Virginia, American Physical Therapy Association (APTA); 2014. (Acceso 24 de junio de 2015). Recuperado a partir de: http://www.capteonline.org/uploadedFiles/CAPTEorg/ About_CAPTE/Resources/Accreditation_Handbook/EvaluativeCriteria_PT.

5. Health and Care Professions Council (HCPC). Standards of proficiency. Physiotherapists. 2013. (Acceso junio 24 de 2015). Recuperado a partir de: http://www.hpc-uk.org/ assets/documents/10000DBCStandards_of_Proficiency_ Physiotherapists.pdf.

6. European Region of World Confederation for Physical Therapy (WCPT). European Physiotherapy Benchmark Statement. Brussels, Belgium: ER-5WCPT; 2003. (Acceso junio 24 de 2015). Recuperado a partir de: http://www. erwcpt.eu/education/documents_relevant_to_education.

7. Australian Physiotherapy Council. Australian Standards for Physiotherapy. Safe and Effective Physiotherapy. Julio 2006. (Acceso junio 26 de 2015). Recuperado a partir de: https://physiocouncil.com.au/media/1021/the-australian-standards-for-physiotherapy-2006.pdf

8. The World Health Professions Alliance WHPA. Statement on Interprofessional Collaborative Practice (Acceso junio 26 de 2015). Recuperado a partir de: http://www.whpa. org/WHPa_statement_collaborative_practice.pdf.

9. World Health Organization (WHO). Health Professions Network Nursing and Midwifery Office within the Department of Human Resources for Health. Framework for Action on Interprofessional Education and Collaborative Practice (Acceso junio 27 de 2015). Recuperado a partir de: http://apps.who.int/iris/bitstream/10665/70185/1/ WHO_HRH_HPN_10.3_eng.pdf. 
10. Interprofessional Education Collaborative Expert Panel. (2011). Core competencies for interprofessional collaborative practice: Report of an expert panel. Washington, D.C. Interprofessional Education Collaborative. (Acceso junio 27 de 2015). Recuperado a partir de: http://www. aacn.nche.edu/education-resources/ipecreport.pdf.

11. Organización Panamericana de la Salud (OPS). Hacia una década de recursos humanos en salud para las Américas. Llamado a la acción de Toronto. 2006-2015. (Acceso 10 de julio de 2015). Recuperado a partir de: http://www. observatoriorh.org/sites/default/files/webfiles/fulltext/ OPS_desafios_toronto_2005.pdf

12. Organización Mundial de la Salud (OMS), Oficina Regional para las Américas. Organización Panamericana de la Salud. Construyendo una nueva agenda de recursos humanos para la salud. Los equipos de salud ante nuevos y renovados desafios. Documento de trabajo Reunión de Recursos humanos. (Acceso 10 de julio de 2015). Recuperado a partir de: http://www.observatoriorh.org/sites/ default/files/webfiles/fulltext/2015/reu_rhs_sept_arg/ doc_trabajo_reu_arg.pdf.

13. República de Colombia. Ley 1164 de 2007. Por la cual se dictan disposiciones en materia del Talento Humano en Salud. Congreso de la República de Colombia.

14. República de Colombia. Ley 528 de 1.999. Reglamentación del ejercicio de la profesión de fisioterapia. Normas en materia de ética profesional y otras disposiciones. Congreso de la República de Colombia.

15. Academia Nacional de Medicina, Comisión de Educación. Ministerio de la Protección Social, Dirección de Análisis y Política de Recursos Humanos. Grupo interinstitucional para la elaboración de propuestas de perfiles y competencias profesionales básicas en salud. Guía Metodológica Número 2 "Aproximación metodológica para la descripción de competencias profesionales básicas en salud". Documento de trabajo. (Acceso agosto 10 de 2015). Recuperado a partir de: https://www.minsalud.gov.co/ salud/Documents/Observatorio\%20Talento\%20Humano\%20en\%20Salud/3-ReferentesNormativos\%20.pdf.

16. Asociación Colombiana de Fisioterapia (ASCOFI). Asociación Colombiana de Facultades de Fisioterapia (ASCOFAFI), Colegio Colombiano de Fisioterapeutas (COLFI). Asociación Colombiana de Estudiantes de Fisioterapia
(ACEFIT). Perfil profesional y competencias del fisioterapeuta en Colombia. Bogotá, mayo de 2015. (Acceso agosto 10 de 2015). Recuperado a partir de: https://www. minsalud.gov.co/sites/rid/Lists/BibliotecaDigital/RIDE/ VS/TH/Perfil-profesional-competencias-FisioterapeutaColombia.pdf.

17. Universidad Industrial de Santander. Facultad de Salud. Escuela de Fisioterapia. Proyecto Educativo Programa de Fisioterapia. Bucaramanga 2005.

18. Universidad Industrial de Santander. Facultad de Salud. Escuela de Fisioterapia. Proyecto Educativo Programa de Fisioterapia. Bucaramanga 2014.

19. Universidad de Boyacá. Facultad de Ciencias de la Salud. Proyecto Educativo del Programa de Fisioterapia. (Acceso agosto 12 de 2015). Recuperado a partir de: file:///C:/ Users/Usuario/Downloads/PEP_Fisioterapia\%20(2).pdf

20. Silva SR, Castellanos R. Conceptualización de las prácticas formativas del Programa de Fisioterapia de la Universidad de Boyacá. Congreso de Investigación y Pedagogía. (Acceso agosto 12 de 2015). Recuperado a partir de: http://tics.uptc.edu.co/eventos/index.php/cong_inv_pedagogia/con_inv_pedag/paper/viewFile/74/74.

21. Universidad Nacional de Colombia. Proyecto Educativo de Programa. Autoevaluación y seguimiento de la calidad de los programas de pregrado. Fisioterapia. Bogotá 2013. (Acceso agosto 12 de 2015). Recuperado a partir de: http://www.pregrado.unal.edu.co/docs/pep/pep_2_20. pdf

22. República de Colombia. Ley 1438 de 2011. Por medio de la cual se reforma el Sistema General de Seguridad en Salud y se dictan otras disposiciones. Congreso de la República de Colombia.

\section{Correo electrónico de los autores}

Aminta Casas: ascasas@uis.edu.co Paula C.Ramírez: pcramirez@uis.edu.co 